\section{Tricornered/NDR kinase signaling mediates PINK1-directed mitochondrial quality control and tissue maintenance}

\author{
Zhihao Wu, ${ }^{1}$ Tomoyo Sawada, ${ }^{2,3}$ Kahori Shiba, ${ }^{4}$
} Song Liu, ${ }^{1}$ Tomoko Kanao, ${ }^{5}$ Ryosuke Takahashi, ${ }^{2,3}$ Nobutaka Hattori, ${ }^{4,5,6}$ Yuzuru Imai, ${ }^{6,7}$ and Bingwei $\mathrm{Lu}^{1,7}$

${ }^{1}$ Department of Pathology, Stanford University School of Medicine, Stanford, California 94305, USA; ${ }^{2}$ Department of Neurology, Graduate School of Medicine, Kyoto University, Kyoto 606-8507, Japan; ${ }^{3}$ CREST (Core Research for Evolutionary Science and Technology), Japan Science and Technology Agency, Saitama 332-0012, Japan; ${ }^{4}$ Department of Neurology, Juntendo University Graduate School of Medicine, Tokyo 113-8421, Japan; ${ }^{5}$ Research Institute for Diseases of Old Age, Juntendo University Graduate School of Medicine, Tokyo 113-8421, Japan; ${ }^{6}$ Department of Neuroscience for Neurodegenerative Disorders, Juntendo University Graduate School of Medicine, Tokyo 113-8421, Japan

Eukaryotes employ elaborate mitochondrial quality control (MQC) to maintain the function of the power-generating organelle. Parkinson's disease-associated PINK1 and Parkin actively participate in MQC. However, the signaling events involved are largely unknown. Here we show that mechanistic target of rapamycin 2 (mTORC2) and Tricornered (Trc) kinases act downstream from PINK1 to regulate MQC. Tre is phosphorylated in mTORC2dependent and mTORC2-independent manners and is specifically localized to mitochondria in response to PINK1, which regulates mTORC2 through mitochondrial complex-I activity. Genetically, mTORC2 and Trc act upstream of Parkin. Thus, multiplex kinase signaling is acting between PINK1 and Parkin to regulate MQC, a process highly conserved in mammals.

Supplemental material is available for this article.

Received August 13, 2012; revised version accepted December 4, 2012.

To sustain energy supply and overall cellular health, elaborate quality control systems are deployed to maintain mitochondrial integrity and functionality (Narendra and Youle 2011; Rugarli and Langer 2012). Mitochondrial quality control (MQC) is particularly important for the maintenance of neural and muscular tissues. PINK1

[Keywords: Tricornered; NDR; mTORC2; PINK1; mitochondrial quality control]

${ }^{7}$ Corresponding authors

E-mail bingwei@stanford.edu

E-mail yzimai@juntendo.ac.jp

Article is online at http://www.genesdev.org/cgi/doi/10.1101/gad.203406.112.
(Valente et al. 2004) and Parkin (Kitada et al. 1998), two proteins associated with familial Parkinson's disease (PD), have been identified as central players in a cellular pathway that directs MQC, a dynamic and multifaceted network encompassing mitochondrial complex-I (CI) function (Liu et al. 2011; Vilain et al. 2012), fission/fusion dynamics (Yang et al. 2008), transport (Wang et al. 2011; Liu et al. 2012), and mitophagy (Liu and Lu 2010; Narendra et al. 2010). Genetic epistasis studies, first in Drosophila (Clark et al. 2006; Park et al. 2006; Yang et al. 2006) and later in mammalian cells (Exner et al. 2007), positioned PINK1 upstream of Parkin in MQC. Studies in mammalian cells have further shown that PINK1 becomes stabilized on the outer membrane of depolarized mitochondria, where it recruits Parkin from the cytosol to promote mitophagy (Narendra et al. 2010). However, the mechanisms of PINK1 and Parkin action and the signaling events involved in the MQC process are poorly understood, with various discordant models having been proposed (Narendra and Youle 2011).

We continued using the powerful tools available in Drosophila to dissect the genetic program underlying PINK1/Parkin-directed MQC. Here we identify mechanistic target of rapamycin 2 (mTORC2) and tricornered (Trc) kinase signaling as intermediate steps between PINK1 and Parkin in the MQC process in Drosophila and further verify the findings in mammalian cells. Our results reveal a previously unanticipated complexity of signaling events involved in PINK1/Parkin-directed MQC and tissue maintenance and shed new light on how mTORC2 and Trc, two important kinases critically involved in cellular morphogenesis, development, growth, and disease, are regulated in an in vivo setting.

\section{Results and Discussion}

To elucidate the signaling events acting downstream from PINK1, we performed genetic screens as described before (Liu and Lu 2010; Liu et al. 2012) to identify genes that can modify PINK1 loss-of-function (LOF)-induced wing posture and flight ability defects. This led to the identification of mTORC2 components as strong modifiers of PINK1. While overexpression of the mTORC2 components Rictor and Sin1 (Hietakangas and Cohen 2007), either individually or in combination, had no obvious effects on their own (Supplemental Fig. S1), they effectively suppressed dPINK1 LOF phenotypes with respect to the integrity and function of flight muscle, as measured by thoracic ATP level and wing posture (Fig. 1A,B;Supplemental Fig. S1D) and the loss of dopaminergic neurons (DNs) in the PPL1 cluster (Fig. 1E). Conversely, rictor or sin1 mutations significantly enhanced $d P I N K 1$ LOF phenotypes (Fig. 1A-E; Supplemental Fig. S1D), although the rictor or sin 1 mutant alone did not show obvious phenotypes in these assays (Supplemental Fig. S1A-C). The effects of mTORC2 components on tissue integrity correlated well with the effects on mitochondrial morphology, with mTORC2 gain of function (GOF) rescuing the mitochondrial aggregation phenotype caused by dPINK1 LOF, whereas mTORC2 LOF had opposite effects (Fig. 1C,D).

We next explored the molecular mechanisms underlying the strong genetic interaction between PINK1 and mTORC2. Using phosphorylation of Drosophila AKT at S505 as readout of mTORC2 activity (Sarbassov et al. 


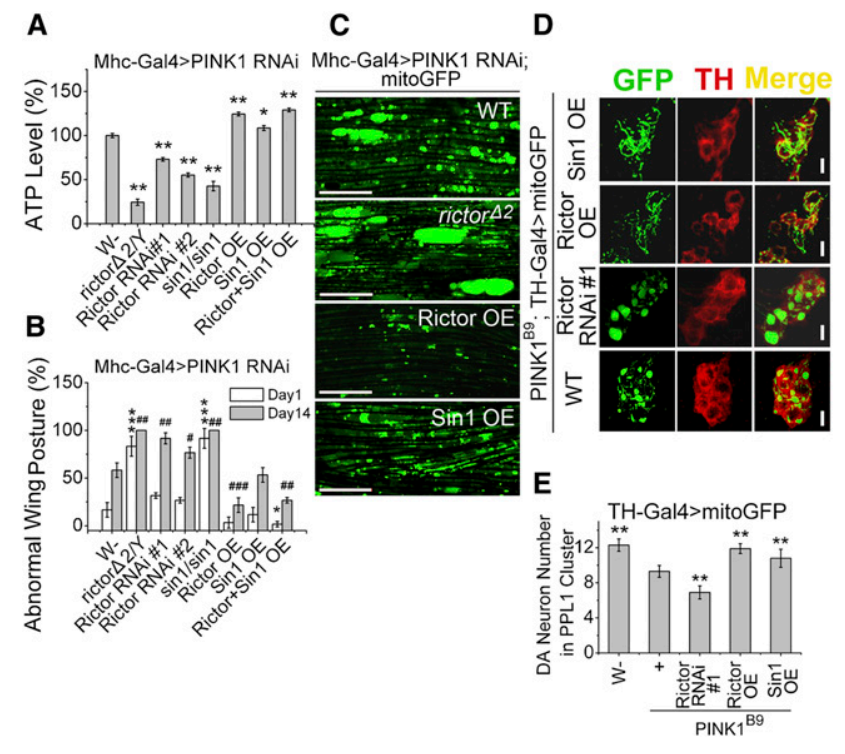

Figure 1. Genetic interaction between PINK1 and mTORC2. (A) PINK1 RNAi-induced ATP level drop was rescued by Rictor or Sin 1 overexpression (OE) but enhanced by their LOF. (B) PINK1 RNAi-induced abnormal wing posture was rescued by Rictor overexpression or Rictor + Sin 1 overexpression but enhanced by their LOF. (C) PINK1 RNAi-induced mitochondrial aggregation in muscle was strongly rescued by Rictor overexpression and moderately rescued by Sin 1 overexpression but was enhanced by rictor LOF. Mitochondrial morphology was monitored with a mito-GFP reporter. Bar, $30 \mu \mathrm{m}$. (D) PINK1 LOF-induced mitochondrial aggregation in DNs was strongly rescued by Rictor overexpression and moderately rescued by Sin 1 overexpression but enhanced by rictor LOF. Bar, $5 \mu \mathrm{m}$. (E) PINK1 LOF-induced loss of DNs in the PPL1 cluster was rescued by Rictor overexpression but enhanced by rictor LOF. A rictor deletion mutant $(\Delta 2)$ and two independent rictor RNAi lines $(\# 1$ and \#2) were used in LOF studies. ( ${ }^{\star}$ or $\left.{ }^{\#}\right) P<0.05$; ( ${ }^{\star \star}$ or ${ }^{\# \#)} P<0.01$; $\left(^{\star \star \star}\right.$ or ${ }^{\# \# \#) ~} P<0.005$ in one-way ANOVA tests when data from day 1 or day 14 were compared.

2005), we found that the p-S505-AKT level was significantly reduced in the dPINK1 LOF condition but increased by PINK1 GOF (Fig. 2A), indicating that PINK1 regulates $\mathrm{mTORC} 2$ activity in vivo. To further validate this point, we affinity-purified Drosophila mTORC2 from transgenic animals expressing a Flag-tagged Sin1 (Koike-Kumagai et al. 2009). In vitro kinase assays using purified mTORC2 as the kinase and kinase-dead (KD) GST-AKT as the substrate showed that the specific activity of mTORC2 was reduced in the $d P I N K 1^{\mathrm{BP}}$-null mutant but increased in PINK1 GOF background (Fig. 2B). The amounts of dTOR copurifying with Flag-Sinl were comparable among the various genotypes (Fig. 2B), suggesting that PINK1 primarily regulates mTORC2 kinase activity instead of its assembly. Consistent with impaired mTORC2 signaling contributing to PINK1 pathogenesis, phosphatidylinositol 3,4,5-trisphosphate $\left(\mathrm{PIP}_{3}\right)$ treatment, which could directly activate mTORC2 in mammalian cells (Gan et al. 2011), partially restored mTORC2 activity (Fig. 2C,D) and rescued dPINK1 mutant phenotypes in terms of lifespan, wing posture, and mitochondrial morphology (Fig. 2E-G).

Despite the importance of mTORC2 in physiology and disease, little is known about how it is regulated by upstream signals (Zoncu et al. 2011). We further investigated how PINK1 regulates mTORC2 activity. Given that PINK1 is a mitochondrial resident protein intimately linked to CI function and that CI dysfunction could phenocopy dPINK1 LOF effects (Supplemental Fig. S2A-C), we reasoned that the functional status of $\mathrm{CI}$ might affect mTORC2 activity. To test this hypothesis, we first examined p-S505-AKT levels in wild-type animals treated with the CI inhibitor rotenone (Fig. $2 \mathrm{H}$ ) or with CI subunits knocked down by RNAi (Fig. 2I). These manipulations led to decreased p-S505-AKT levels. Consistently, mTORC2 purified from CI-impaired animals exhibited reduced kinase activity in vitro (Supplemental Fig. S2D). Importantly, in dPINK1 mutants whose CI activity was supplemented by yeast $\mathrm{NADH}$ ubiquinone oxidoreductase (yNDI1), the mutant phenotypes were effectively rescued (Supplemental Fig. S2E-G), consistent with a recent study (Vilain et al. 2012), and p-S505-AKT level was restored to normal (Fig. 2I). Further supporting a functional link between CI and mTORC2 activation, mTORC2 LOF animals were particularly sensitive to rotenone treatment compared with other stress treatments (Supplemental Fig. S3A-F), and LOF of mTORC2 and CI synergistically disrupted muscle function (Supplemental Fig. S3G). These results demonstrate that the functional state of CI maintained by PINK1 is a critical determinant of mTORC2 activity. Supporting this notion, PINK1 was found to associate with components of CI (Supplemental Fig. S2H).

Next, we sought to determine the signaling events downstream from mTORC2 that mediate PINK1-directed MQC and tissue maintenance. Surprisingly, even though AKT is considered a key downstream target of mTORC2 (Wullschleger et al. 2006; Russell et al. 2011; Zoncu et al. 2011) and AKT function is compromised in the dPINK1 mutant, as shown by a reduced p-S505-AKT level, overexpression of wild-type or phospho-mutant forms of AKT had no obvious effect on dPINK1 LOF phenotypes in terms of mitochondrial morphology and tissue maintenance (Supplemental Fig. S4A-C). Knocking down AKT by RNAi also had no obvious effect (Supplemental Fig. $\mathrm{S} 4 \mathrm{~A}-\mathrm{C}$ ). Thus, the best-studied mTORC2 target, AKT, is unlikely to be a key mediator of mTORC2 function in PINK1-controlled MQC and tissue maintenance.

Trc kinase was recently identified as a downstream effector of mTORC2 in regulating the dendritic tiling of Drosophila sensory neurons (Koike-Kumagai et al. 2009). Trc was originally isolated in a screen for genes affecting wing hair morphogenesis (Geng et al. 2000) and was later found to be important for dendritic patterning (Emoto et al. 2004) in Drosophila. Trc is homologous to mammalian nuclear Dbf-2-related (NDR) kinases (Hergovich et al. 2006). Whether Trc/NDR regulates mitochondrial function or neuromuscular tissue maintenance is not known. Interestingly, although overexpression of Trc LOF or GOF transgenes alone had little effect on muscle function (Supplemental Fig. S5A-C), Trc GOF transgenes effectively suppressed $d P I N K 1$ LOF phenotypes in muscle (Fig. 3A,B; Supplemental Fig. S5D) or DN (Fig. 3E) maintenance and mitochondrial morphology (Fig. 3C,D), whereas dominant-negative Trc (Trc-K122A or Trc-K122/ T453A) had opposite effects (Fig. 3A-E; Supplemental Fig. S5D). Note that wild-type or constitutively active Trc (Trc-S292E and Trc-T453E) were equally effective in the GOF assays, indicating that overexpressed wild-type Trc became sufficiently activated, as indicated by its high level phosphorylation at S292 (Fig. 3F).

Consistent with Trc acting downstream from mTORC2, the enhancement of $d P I N K 1$ LOF phenotypes by rictor mutation was attenuated by wild-type Trc (Trc-WT) or Trc-S292E GOF transgenes (Supplemental Fig. S6A,C), 
A

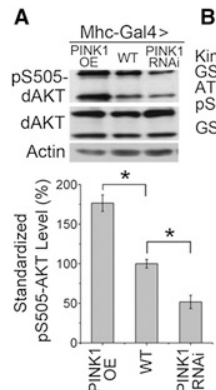

E
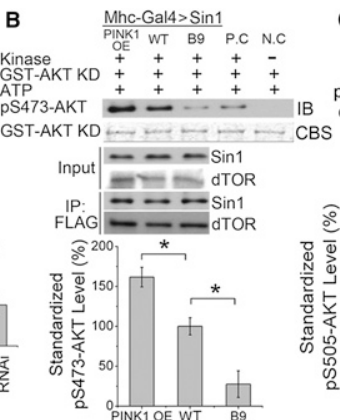

C
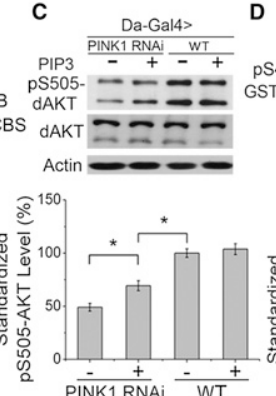

G

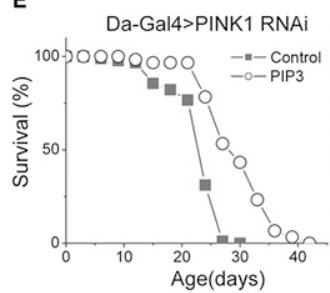

$\mathbf{F}$

Da-Gal4>PINK1 RNAi
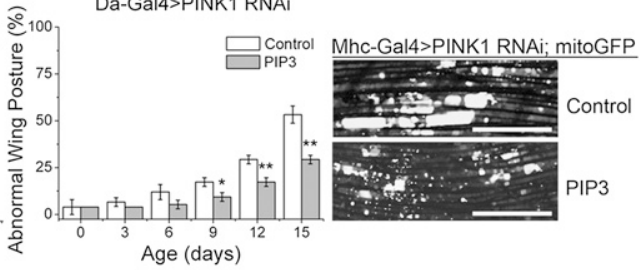

H

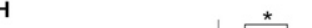

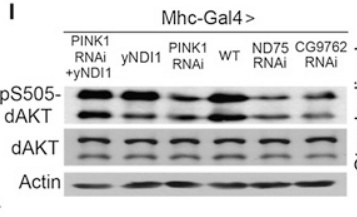

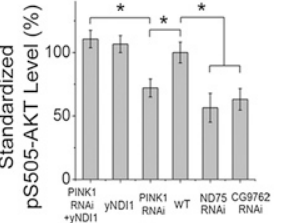

Figure 2. Effects of PINK1 and mitochondrial CI function on TORC2 activity. (A) Western blot analysis of $M h c$-Gal4-driven control (wild type [WT]), PINK1 overexpression, and PINK1-RNAi muscle extracts with the indicated antibodies and data quantification. $(B, t o p)$ In vitro phosphorylation of recombinant human GST-AKT-KD by Drosophila TORC2 affinity-purified from control, PINK1overexpressing, and PINK1 LOF animals, all expressing a Sin1-Flag transgene. (Bottom) Similar amounts of Sin 1 and dTOR were present in the extracts or immunoprecipitated TORC2. After kinase reaction, AKT phosphorylation was detected with anti-pS473-AKT, and total GST-AKT-KD was detected by Coomassie Blue staining (CBS). (P.C.) Positive control with extract added; (N.C.) negative control with no kinase added. Bar graph shows data quantification. $(C-G)$ The rescuing effect of PIP3 treatment on in vivo p-S505-AKT level $(C)$, in vitro mTORC2 kinase activity $(D)$, lifespan $(E)$, wing posture $(F)$, and muscle mitochondrial morphology $(G)$ in PINK1-RNAi animals. Values represent relative protein amounts after normalization with controls. Mitochondrial morphology was monitored with a mito-GFP reporter. In vitro mTORC2 kinase assay was performed as in $B$. Bar graphs show data quantification. Bar, $30 \mu \mathrm{m} .(H)$ Reduction of pS505AKT level by rotenone treatment of wild-type animals. (I) Effect of CI subunit (ND75 and CG9762) RNAi on pS505-AKT level and restoration of the p-S505-AKT level in PINK1-RNAi animals after yNDIl coexpression. Values represent relative protein amounts after normalization with controls. Bar graphs show data quantification. $\left.{ }^{\star}\right) P<0.05$ in one-way ANOVA or Student's $t$-tests.

whereas the rescue of $d P I N K 1$ LOF phenotypes by Rictor overexpression was fully blocked by Trc LOF transgenes (Supplemental Fig. S6B,D). Moreover, Trc RNAi induced mitochondrial aggregation and DN loss in a wild-type background (Supplemental Fig. S7A-C). These results thus establish Trc as a key component of the PINK1 pathway in MQC and tissue maintenance.

We further explored the biochemical mechanism of the signaling events involving PINK1, mTORC2, and Trc. Consistent with Trc acting downstream from PINK1, phosphorylation of Trc at S292 and T453, which correlate with Trc activation (Emoto et al. 2006), were reduced in dPINK1 mutants but significantly increased in PINK1 GOF flies (Fig. 3F). This was further supported by in vitro assays of kinase activities of Trc purified from PINK1 LOF and GOF flies using phosphorylation of AAK1, a newly identified substrate of mammalian NDR (Ultanir et al. 2012), as readout (Fig. 3G). Interestingly, the effect of PINK1 on T453 site phosphorylation was mTORC2-dependent, whereas its effect on S292 was mTORC2-independent (Fig. 3H), suggesting that PINK1 regulates these two phosphorylation events through distinct mechanisms. We next tested whether activated Trc is localized to mitochondria to exert its effects on MQC. Strikingly, p-T453-Trc and p-S292-Trc were found primarily in the mitochondrial fraction, whereas total Trc was found in both the cytoplasmic and mitochondrial fractions (Supplemental Fig. S8A). Since mTORC2 is also localized to mitochondria (Supplemental Fig. S8B), mitochondria may serve as a novel platform for mTORC2 and Trc signaling.

Parkin plays a critical role in MQC and maintenance of muscle and DNs and acts downstream from PINK1. We found that the LOF effects of Rictor (Fig. 4A,E) or Trc (Fig. 4C,G) in enhancing dPINK1 mutant phenotypes were completely blocked by Parkin GOF, suggesting that Parkin acts downstream from TORC2 and Trc in the PINK1 pathway. Consistently, Rictor GOF (Fig. $4 \mathrm{~B}, \mathrm{~F}$ ) or Trc GOF (Fig. 4D,H) failed to rescue the abnormal wing posture and mitochondrial morphology phenotypes of the parkin mutant. Next, we tested the genetic interaction between mTORC2/Trc and key executors of MQC known to genetically interact with PINK1, including the mitochondrial fusion protein Marf (Liu and $\mathrm{Lu}$ 2010; Ziviani et al. 2010), the autophagy regulator Atg1 (Liu and Lu 2010), and the mitochondrial transport protein Miro (Wang et al. 2011; Liu et al. 2012). Marf RNAi or Atg1 GOF effectively blocked the enhancing effects of mTORC2 LOF (Fig. 4I,J) or Trc LOF (Fig. 4K,L) in the dPINK1 mutant background. Miro RNAi was also effective, although to a lesser degree (Fig. 4I-L). Together, these genetic epistasis data support critical roles of $\mathrm{mTORC} 2$ and Trc in mediating the effect of PINK1 in MQC and tissue maintenance.

In mammalian cells, the PINK1-Parkin pathway also plays central roles in MQC, with Parkin recruited to damaged mitochondria in a PINK1-dependent manner to promote mitophagy (Narendra et al. 2010). We tested whether the mammalian Trc homologs NDR1/2 act in PINK1/Parkin-directed MQC. Knockdown of NDR1 but not NDR2 in HeLa cells led to altered mitochondrial distribution, compromised recruitment of Parkin by PINK1, and delayed clearance of damaged mitochondria (Supplemental Fig. S9A-C). The mitophagy defects induced by NDR1 RNAi were rescued by a siRNA-resistant NDR1 construct (Supplemental Fig. S9B,D). Importantly, we detected reduced phosphorylation of NDR1 at the corresponding T444 site in PINK1 ${ }^{-/-}$mouse embryonic fibroblasts (MEFs) expressing a kinase-dead form of PINK1 but not wild-type PINK1 (Supplemental Fig. S10). Due to the hypersensitivity of $\mathrm{p}$-NDR1 to endogenous phosphatase activities as previously reported (Koike-Kumagai et al. 2009), treatment with okadaic acid (OA) was needed to reveal the effect of PINK1 on the p-NDR1 level. These results support that NDR1 acts in the PINK1/Parkin pathway in mammals. In carbonyl cyanide m-chlorophe- 


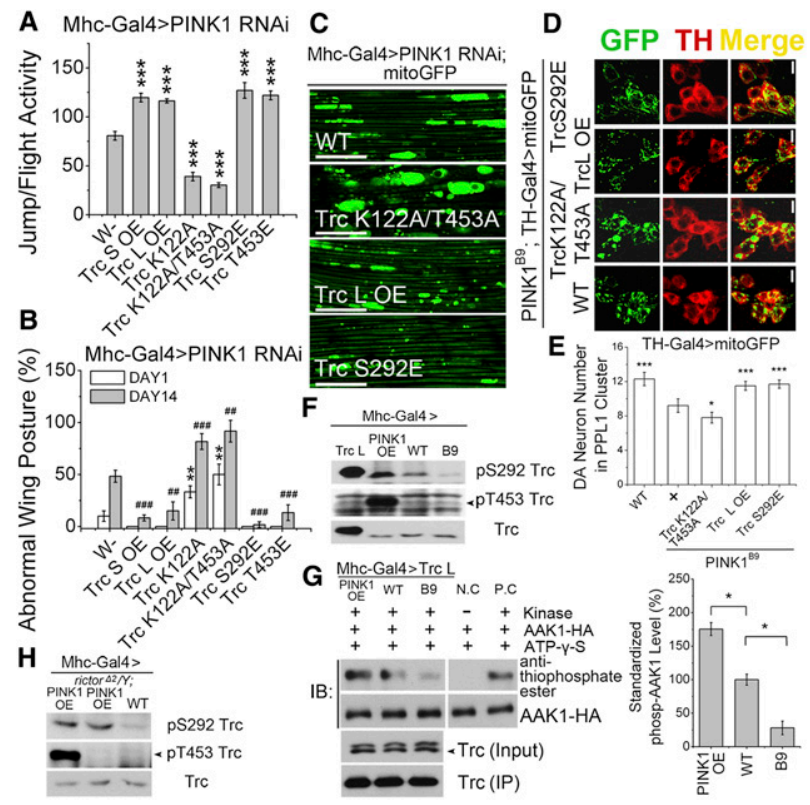

Figure 3. Evidence that Tre acts in the PINK1 pathway. $(A, B) M h c$ Gal4>PINK1 RNAi-induced flight ability $(A)$ and wing posture $(B)$ defects were rescued by the coexpression of the short (S) or long (L) isoforms of wild-type Trc or constitutively active (S292E or T453E) Trc but enhanced by dominant-negative (K122A or K122A/T453A) Trc. $\left({ }^{\star}\right.$ or $\left.{ }^{\#}\right) P<0.05$; $\left({ }^{\star \star}\right.$ or $\left.{ }^{\# \#}\right) P<0.01$; $\left(^{\star \star \star}\right.$ or ${ }^{\# \# \#) ~} P<0.005$ in oneway ANOVA tests when data from day $1\left(^{\star}\right)$ or day $14\left(^{\#)}\right.$ were compared. (C) Mhc-Gal4>PINK1 RNAi-induced mitochondrial aggregation in indirect flight muscle was rescued by the coexpression of Trc-L or Trc-S292E but enhanced by Trc-K122A/T453A. Mitochondrial morphology was monitored with a mito-GFP reporter. Bar, $30 \mu \mathrm{m}$. $(D, E) P I N K 1^{B 9}$ mutation-induced mitochondrial aggregation in DNs $(D)$ or loss of DNs in the PPL1 clusters $(E)$ was strongly rescued by $\mathrm{TH}$-Gal4-driven expression of wild-type or constitutively active Trc but enhanced by dominant-negative Trc. Bar, $5 \mu \mathrm{m}$. (F) Western blot analysis showing the effects of PINK1 LOF or overexpression on Trc phosphorylation. Trc-L overexpression served as a positive control. $(G)$ In vitro kinase assay showing the effects of PINK1 LOF or overexpression on Trc kinase activity. Trc-L purified from control, PINK1 mutant, and PINK1-overexpressing animals was tested for kinase activity using AAK1-HA as a substrate in the presence of ATP- $\gamma-S$ and phosphorylation detected by anti-thiophosphate ester antibody after esterification with p-nitrobenzylmesylate (PNBM). (P.C.) Positive control with extract added; (N.C.) negative control with no kinase added. Bar graph shows data quantification. $(H)$ Western blot analysis showing that PINK1-induced Trc phosphorylation of T453 was Rictor-dependent, whereas that of S292 was Rictor-independent. $\left(^{\star}\right) P<0.05 ;\left(^{\star \star \star}\right) P<0.005$ in one-way ANOVA tests in $E$ and $G$.

nylhydrazone (CCCP)-treated HeLa cells, NDR1 was found to colocalize with Parkin and the mitochondrial outer membrane marker Tom 20 (Supplemental Fig. S9B), suggesting that NDR1 might also be localized to mitochondria to exert MQC. In Parkin stably transfected HeLa cells that were subjected to Rictor RNAi (Supplemental Fig. S11A,D) or in rictor $^{-/-}$MEF cells (Supplemental Fig. S11B,C,E), the recruitment of Parkin to damaged mitochondria and the kinetics of mitophagy were also delayed, supporting a critical role of mTORC2 in PINK1/Parkindirected mitophagy in mammals.

We further investigated the mechanism of action of NDR1 in mammalian cells. It has been reported that Parkin is phosphorylated and activated by phosphoryla- tion upon CCCP treatment (Kondapalli et al. 2012; ShibaFukushima et al. 2012). In HeLa cells stably transfected with Parkin, CCCP treatment led to increased phosphorylation of NDR1 and Parkin as detected with Phos tag. NDR1 RNAi significantly attenuated Parkin phosphorylation (Supplemental Fig. S12A), consistent with Parkin acting downstream from NDR1. Further supporting this notion, the activity of Parkin as measured by its autoubiquitination was reduced by NDR1 RNAi (Supplemental Fig. S12B). Moreover, the destabilization of MQC-related proteins Mfn1 and Miro1 by CCCP-activated PINK1/ Parkin signaling was significantly attenuated by NDR1 RNAi (Supplemental Fig. S12A). These results support the notion that, as in flies, NDR1 kinase signaling acts upstream of Parkin and the other key MQC players in the mammalian MQC pathway.

Our results demonstrate that $\mathrm{mTORC} 2$ and Trc signaling are actively involved in the dynamic MQC network directed by PINK1 and that they act between PINK1 and Parkin in a conserved signaling pathway. This finding reveals a previously unappreciated complexity in the signaling steps between PINK1 and Parkin. This is the first time both mTORC2 and Trc are directly implicated in MQC. The fact that PINK1 regulates Trc T453 phosphorylation in an mTORC2-dependent manner and S292 phosphorylation in an mTORC2-independent manner suggests the involvement of additional kinases in this MQC pathway. Our finding of active p-Trc localizing primarily to mitochondria suggests that mitochondria may serve as a key platform for Trc signaling. The kinase that directly acts on Trc T453 in the context studied here remains to be identified. Although mTORC2 is required, it may not be the kinase that directly phosphorylates this site (Koike-Kumagai et al. 2009). Similarly, although PINK1 is required for mTORC2 activation, our results suggest that PINK1 acts through maintaining mitochondrial CI activity to influence mTORC2 activity, rather than directly phosphorylating $\mathrm{mTORC} 2$ as proposed in a previous study (Murata et al. 2011). Our findings offer new insights into the novel role of mitochondria in regulating $\mathrm{mTORC} 2$ and Trc/ NDR kinase signaling.

mTORC2 and Trc signaling both induce cell morphology changes through actin cytoskeleton regulation (Jacinto et al. 2004; Fang and Adler 2010). Given the dynamic changes in mitochondrial distribution and morphology during MQC and the implicated roles of the actin cytoskeleton in regulating mitochondrial distribution and morphology (Boldogh and Pon 2006), it is possible that mTORC2/Trc may act in MQC through cytoskeletal regulation, although this remains to be tested. Our results support the notion that Trc signaling directly impinges on Parkin or the other key MQC executors (Supplemental Fig. S12C). A corollary of our finding is that mitochondrial localization of the kinases and the ensuing MQC may participate in other physiological processes regulated by mTORC2/Trc signaling. Given that deregulated mTORC2 (Zoncu et al. 2011), Trc/NDR (Cornils et al. 2011), and PINK1 and Parkin (Devine et al. 2011) signaling have all been linked to cancer in humans, another important implication of this study is that aberrant MQC signaling also contributes to cancerous growth and that therapeutic agents targeting the newly identified, highly conserved MQC signaling pathway may have broad therapeutic applications. 


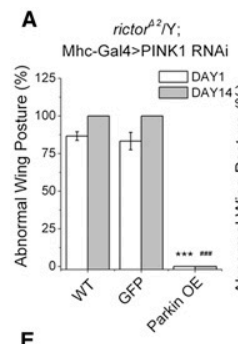

E

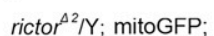

rictor $/ Y$; mitoGFP;
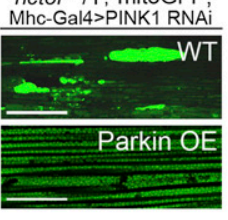

I rictor ${ }^{\mathrm{2}} \mathrm{Y}$;

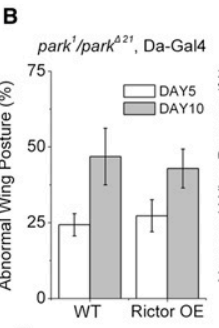

$\mathbf{F}$
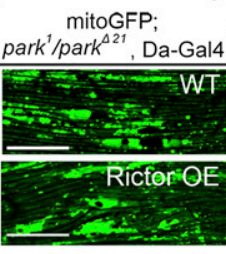

$\mathbf{J}$

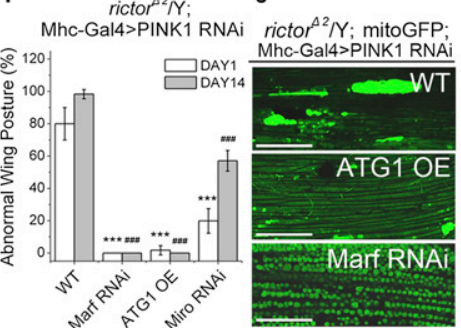

Mhc-Gal4>PINK1 RNAi

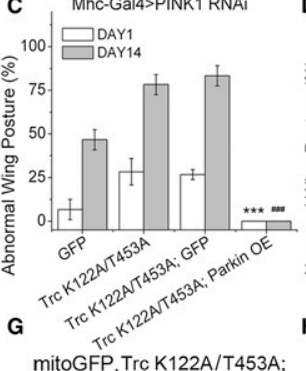

Dark' 1 park ${ }^{21}$, Da-Gal4

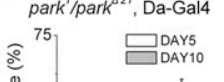

3 times in lysis buffer and twice with the TORC2 kinase buffer (25 mM HEPES at $\mathrm{pH} 7.5,100 \mathrm{mM}$ KAc, $2 \mathrm{mM} \mathrm{MgCl}_{2}$ ). The integrity of the TORC2 was confirmed by Western blot analysis.

For kinase assays, TORC2 immunocomplexes were incubated with $500 \mathrm{ng}$ of human GST-AKT-KD fusion protein in $30 \mu \mathrm{L}$ of TORC2 kinase buffer containing $500 \mu \mathrm{M}$ ATP. The reaction was performed for $30 \mathrm{~min}$ at $30^{\circ} \mathrm{C}$ and terminated by the addition of $30 \mu \mathrm{L}$ of $2 \times$ SDS sample buffer. AKT phosphorylation by TORC2 was analyzed by Western blot using the p-T473-AKT antibody (Cell Signaling Technology).

\section{Mitochondrial and cytoplasmic protein fractionation}

For mitochondria purification, we adopted a previous method (Kristian et al. 2006). Briefly, fly tissues were collected and homogenized in homogenization buffer $(210 \mathrm{mM}$ mannitol, $70 \mathrm{mM}$ sucrose, $1 \mathrm{mM}$ EGTA, $10 \mathrm{mM} \beta$-glycerophosphate, $50 \mathrm{mM}$ $\mathrm{NaF}, 1 \mathrm{mM}$ PMSF, $5 \mathrm{mM}$ HEPES at $\mathrm{pH} 7.12$ ). The homogenate was centrifuged at $1500 \mathrm{~g}$ for $5 \mathrm{~min}$, and the supernatant was collected and centrifuged again at $13,000 \mathrm{~g}$ for $17 \mathrm{~min}$. The supernatant was concentrated as a cytoplasmic protein sample, and the pellet was stored for further mitochondrial purification. For mitochondrial purification, the previous pellet was resuspended in $15 \%$ Percoll solution and loaded onto a $15 \%-22 \%-50 \%$ discontinuous Percoll gradient. The gradient was centrifuged at 30,700g for $6 \mathrm{~min}$, and intact mitochondria were recovered from the $22 \%-50 \%$ gradient interface. Mitochondria were washed with homogenization buffer and subjected to SDS-PAGE.

\section{Quantitative Western blot analysis}

Figure 4. Genetic evidence that Parkin and MQC executors act downstream from TORC2 and Trc in the PINK1 pathway. $(A, E)$ The enhancement of PINK1 RNAi-induced abnormal wing posture $(A)$ or mitochondrial aggregation $(E)$ by rictor deletion was suppressed by Parkin but not GFP overexpression. $(B, F)$ The rescue of PINK1 RNAi-induced abnormal wing posture $(B)$ or mitochondrial aggregation $(F)$ by Rictor overexpression was blocked by Parkin LOF. $(C, G)$ The enhancement of PINK1 RNAi-induced abnormal wing posture $(C)$ or mitochondrial aggregation $(G)$ by dominant-negative Trc was completely suppressed by Parkin but not GFP overexpression. $(D, H)$ The rescue of PINK1 RNAi-induced abnormal wing posture $(D)$ or mitochondrial aggregation $(H)$ by wild-type or constitutively active Trc was blocked by Parkin LOF. $(I, J)$ The enhancement of PINK1 RNAi-induced abnormal wing posture $(I)$ or mitochondrial aggregation $(J)$ by rictor deletion was suppressed by Miro-RNAi, Marf-RNAi, or Atgl overexpression. $(K, L)$ The enhancement of PINK1 RNAi-induced abnormal wing posture by dominant-negative Trc was suppressed by Miro-RNAi, Marf-RNAi, or Atg1 overexpression. $\left(^{\star}\right.$ or $\left.^{\#}\right) P<0.05 ;\left(^{\star \star}\right.$ or $\left.{ }^{\# \#}\right) P<0.01$; and $\left({ }^{\star \star \star}\right.$ or ${ }^{\# \# \#)} P<0.005$ in one-way ANOVA or Student's $t$-tests when data from day $1\left(^{\star}\right)$ or day $14\left({ }^{\#}\right)$ were compared. Bars: $E-F, J, L, 30 \mu \mathrm{m}$.

To obtain quantitative Western blot results, experimental conditions such as the amounts of protein loaded, the antibody dilutions, and exposure times were adjusted to make sure that the Western blot signals were in the linear range. For the in vitro kinase assays, Western blot analyses were performed using standard protocols. After incubation with the ECL reagent (PerkinElmer, Inc.), the signals were scanned using a Typhoon 9400 system (GE Healthcare Life Sciences), and signal intensity was quantified by ImageQuantTL. For the other immunoblots, the signals were recorded on X-ray film and scanned, and the signal intensity was analyzed by ImageQuantTL or ImageJ. Statistical analyses were based on data from three independent repeats.

\section{Statistical analysis}

Two-tailed Student's $t$-tests were used in the statistical analysis when comparing two samples. A one-way ANOVA test was used when multiple samples were compared.

\section{Materials and methods}

\section{GST fusion protein preparation}

The construct expressing human GST-AKT-KD used as the substrate in the in vitro TORC2 kinase assays was a gift from Dr. Dianqing Wu. To avoid autophosphorylation of AKT, the Lys179 and Thr308 residues were mutated (Gan et al. 2011). GST fusion protein was expressed in the Escherichia coli BL21 (DE3) strain and purified using glutathione-agarose beads following standard protocols.

\section{Immunoprecipitation and kinase assay of TORC2}

For TORC2 immunoprecipitation, methods adopted from previous studies (Koike-Kumagai et al. 2009; Gan et al. 2011) were used. Briefly, flies were crossed and raised in standard medium at $25^{\circ} \mathrm{C}$. Male flies expressing Flag-tagged Sinl were collected and homogenized in lysis buffer $140 \mathrm{mM}$ HEPES at $\mathrm{pH} 7.5,120 \mathrm{mM} \mathrm{NaCl}, 0.3 \%$ CHAPS, $1 \mathrm{mM}$ EDTA, $10 \mathrm{mM}$ $\beta$-glycerophosphate, $50 \mathrm{mM} \mathrm{NaF}, 1 \mathrm{mM}$ PMSF). TORC2 was immunoprecipitated by incubating the supernatant with pre-equilibrated anti-Flag $\mathrm{M} 2$ beads (Sigma Aldrich) for $3 \mathrm{~h}$ at $4^{\circ} \mathrm{C}$. Immunocomplexes were washed

\section{Acknowledgments}

We thank Dr. P. Aspenström, Dr. E. Baehrecke, Dr. R. Baloh, Dr. S. Birman, Dr. J. Chung, Dr. S. Cohen, Dr. K. Emoto, Dr. M. Guo, Dr. Y. Hata, Dr. S. Hatakeyama, Dr. B. Hemmings, Dr. Y.N. Jan, Dr. S. Konur, Dr. M. Magnuson, Dr. N. Matsuda, Dr. K. Nakagawa, Dr. W. Saxton, Dr. K. Tanaka, Dr. G. Thomas, Dr. P. Verstreken, Dr. D. Wu, Dr. K. Zinsmaier, Bloomington Drosophila Stock Center, Vienna Drosophila RNAi Center, and TRiP at Harvard Medical School (NIH/NIGMS R01-GM084947) for flies and reagents, and Lu laboratory members for technical help and discussions. This work was supported by the NIH (R01AR054926 and R01MH080378 to B.L.), Grant-in-Aid for Young Scientists (B) from MEXT in Japan (to Y.I.), the CREST program of JST (to R.T.), a Grant-inAid for Scientific Research on Innovative Areas (to R.T.), and a Grant-inAid for Science Research from the Ministry of Health, Labor, and Welfare in Japan (to R.T.).

\section{References}

Boldogh IR, Pon LA. 2006. Interactions of mitochondria with the actin cytoskeleton. Biochim Biophys Acta 1763: 450-462. 
Clark IE, Dodson MW, Jiang C, Cao JH, Huh JR, Seol JH, Yoo SJ, Hay BA, Guo M. 2006. Drosophila pink1 is required for mitochondrial function and interacts genetically with parkin. Nature 441: 1162-1166.

Cornils H, Kohler RS, Hergovich A, Hemmings BA. 2011. Downstream of human NDR kinases: Impacting on c-myc and $\mathrm{p} 21$ protein stability to control cell cycle progression. Cell Cycle 10: 1897-1904.

Devine MJ, Plun-Favreau H, Wood NW. 2011. Parkinson's disease and cancer: Two wars, one front. Nat Rev Cancer 11: 812-823.

Emoto K, He Y, Ye B, Grueber WB, Adler PN, Jan LY, Jan YN. 2004 Control of dendritic branching and tiling by the Tricornered-kinase/ Furry signaling pathway in Drosophila sensory neurons. Cell 119: 245256.

Emoto K, Parrish JZ, Jan LY, Jan YN. 2006. The tumour suppressor Hippo acts with the NDR kinases in dendritic tiling and maintenance. Nature 443: 210-213.

Exner N, Treske B, Paquet D, Holmström K, Schiesling C, Gispert S, Carballo-Carbajal I, Berg D, Hoepken HH, Gasser T, et al. 2007. Lossof-function of human PINK1 results in mitochondrial pathology and can be rescued by parkin. J Neurosci 27: 12413-12418.

Fang X, Adler PN. 2010. Regulation of cell shape, wing hair initiation and the actin cytoskeleton by Trc/Fry and Wts/Mats complexes. Dev Biol 341: 360-374.

Gan X, Wang J, Su B, Wu D. 2011. Evidence for direct activation of mTORC2 kinase activity by phosphatidylinositol 3,4,5-trisphosphate. J Biol Chem 286: 10998-11002.

Geng W, He B, Wang M, Adler PN. 2000. The tricornered gene, which is required for the integrity of epidermal cell extensions, encodes the Drosophila nuclear DBF2-related kinase. Genetics 156: 1817-1828.

Hergovich A, Stegert MR, Schmitz D, Hemmings BA. 2006. NDR kinases regulate essential cell processes from yeast to humans. Nat Rev Mol Cell Biol 7: 253-264.

Hietakangas V, Cohen SM. 2007. Re-evaluating AKT regulation: Role of TOR complex 2 in tissue growth. Genes Dev 21: 632-637.

Jacinto E, Loewith R, Schmidt A, Lin S, Ruegg MA, Hall A, Hall MN. 2004. Mammalian TOR complex 2 controls the actin cytoskeleton and is rapamycin insensitive. Nat Cell Biol 6: 1122-1128.

Kitada T, Asakawa S, Hattori N, Matsumine H, Yamamura Y, Minoshima S, Yokochi M, Mizuno Y, Shimizu N. 1998. Mutations in the parkin gene cause autosomal recessive juvenile parkinsonism. Nature 392: 605-608.

Koike-Kumagai M, Yasunaga K, Morikawa R, Kanamori T, Emoto K. 2009. The target of rapamycin complex 2 controls dendritic tiling of Drosophila sensory neurons through the Tricornered kinase signalling pathway. EMBO I 28: 3879-3892.

Kondapalli C, Kazlauskaite A, Zhang N, Woodroof HI, Campbell DG, Gourlay R, Burchell L, Walden H, Macartney TJ, Deak M, et al. 2012. PINK1 is activated by mitochondrial membrane potential depolarization and stimulates Parkin E3 ligase activity by phosphorylating Serine 65. Open Biol 2: 120080.

Kristian T, Hopkins IB, McKenna MC, Fiskum G. 2006. Isolation of mitochondria with high respiratory control from primary cultures of neurons and astrocytes using nitrogen cavitation. J Neurosci Methods 152: $136-143$.

Liu S, Lu B. 2010. Reduction of protein translation and activation of autophagy protect against PINK1 pathogenesis in Drosophila melanogaster. PLoS Genet 6: e1001237.

Liu W, Acín-Peréz R, Geghman KD, Manfredi G, Lu B, Li C. 2011. Pink1 regulates the oxidative phosphorylation machinery via mitochondrial fission. Proc Natl Acad Sci 108: 12920-12924.

Liu S, Sawada T, Lee S, Yu W, Silverio G, Alapatt P, Millan I, Shen A, Saxton W, Kanao T, et al. 2012. Parkinson's disease-associated kinase PINK1 regulates miro protein level and axonal transport of mitochondria. PLoS Genet 8: e1002537.

Murata H, Sakaguchi M, Jin Y, Sakaguchi Y, Futami J, Yamada H, Kataoka K, Huh NH. 2011. A new cytosolic pathway from a Parkinson diseaseassociated kinase, BRPK/PINK1: Activation of AKT via mTORC2. I Biol Chem 286: 7182-7189.

Narendra DP, Youle RJ. 2011. Targeting mitochondrial dysfunction: Role for PINK1 and Parkin in mitochondrial quality control. Antioxid Redox Signal 14: 1929-1938.

Narendra DP, Jin SM, Tanaka A, Suen DF, Gautier CA, Shen J, Cookson MR, Youle RJ. 2010. PINK1 is selectively stabilized on impaired mitochondria to activate Parkin. PLoS Biol 8: el000298.
Park J, Lee SB, Lee S, Kim Y, Song S, Kim S, Bae E, Kim J, Shong M, Kim JM, et al. 2006. Mitochondrial dysfunction in Drosophila PINK1 mutants is complemented by parkin. Nature 441: 1157-1161.

Rugarli EI, Langer T. 2012. Mitochondrial quality control: A matter of life and death for neurons. EMBO J 31: 1336-1349.

Russell RC, Fang C, Guan KL. 2011. An emerging role for TOR signaling in mammalian tissue and stem cell physiology. Development 138: 3343-3356.

Sarbassov DD, Guertin DA, Ali SM, Sabatini DM. 2005. Phosphorylation and regulation of Akt/PKB by the rictor-mTOR complex. Science 307: 1098-1101.

Shiba-Fukushima K, Imai Y, Yoshida S, Ishihama Y, Kanao T, Sato S, Hattori N. 2012. PINK1-mediated phosphorylation of the Parkin ubiquitin-like domain primes mitochondrial translocation of Parkin and regulates mitophagy. Sci Rep 2: 1002.

Ultanir SK, Hertz NT, Li G, Ge WP, Burlingame AL, Pleasure SI, Shokat KM, Jan LY, Jan YN. 2012. Chemical genetic identification of NDR1/ 2 kinase substrates AAK1 and Rabin8 uncovers their roles in dendrite arborization and spine development. Neuron 73: 1127-1142.

Valente EM, Abou-Sleiman PM, Caputo V, Muqit MM, Harvey K, Gispert S, Ali Z, Del Turco D, Bentivoglio AR, Healy DG, et al. 2004. Hereditary early-onset Parkinson's disease caused by mutations in PINK1. Science 304: 1158-1160.

Vilain S, Esposito G, Haddad D, Schaap O, Dobreva MP, Vos M, Van Meensel S, Morais VA, De Strooper B, Verstreken P. 2012. The yeast complex I equivalent $\mathrm{NADH}$ dehydrogenase rescues pink1 mutants. PLoS Genet 8: e1002456.

Wang X, Winter D, Ashrafi G, Schlehe J, Wong YL, Selkoe D, Rice S, Steen J, Lavoie MJ, Schwarz TL. 2011. PINK1 and Parkin target miro for phosphorylation and degradation to arrest mitochondrial motility. Cell 147: 893-906.

Wullschleger S, Loewith R, Hall MN. 2006. TOR signaling in growth and metabolism. Cell 124: 471-484.

Yang Y, Gehrke S, Imai Y, Huang Z, Ouyang Y, Wang JW, Yang L, Beal MF, Vogel H, Lu B. 2006. Mitochondrial pathology and muscle and dopaminergic neuron degeneration caused by inactivation of Drosophila Pink1 is rescued by Parkin. Proc Natl Acad Sci 103: 1079310798.

Yang Y, Ouyang Y, Yang L, Beal MF, McQuibban A, Vogel H, Lu B. 2008. Pink1 regulates mitochondrial dynamics through interaction with the fission/fusion machinery. Proc Natl Acad Sci 105: 7070-7075.

Ziviani E, Tao RN, Whitworth AJ. 2010. Drosophila parkin requires PINK1 for mitochondrial translocation and ubiquitinates Mitofusin. Proc Natl Acad Sci 107: 5018-5023.

Zoncu R, Efeyan A, Sabatini DM. 2011. mTOR: From growth signal integration to cancer, diabetes and ageing. Nat Rev Mol Cell Biol 12: $21-35$. 


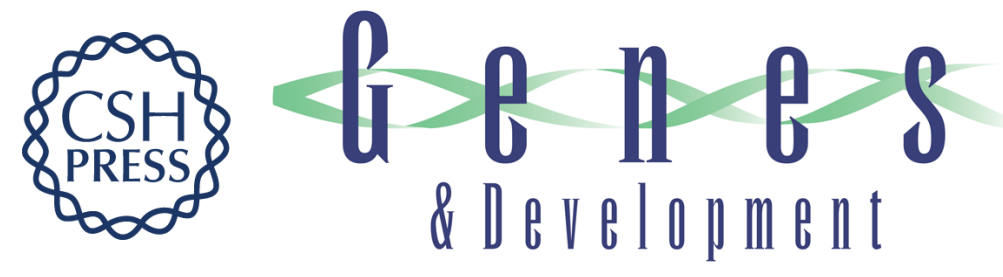

\section{Tricornered/NDR kinase signaling mediates PINK1-directed mitochondrial quality control and tissue maintenance}

Zhihao Wu, Tomoyo Sawada, Kahori Shiba, et al.

Genes Dev. 2013, 27:

Access the most recent version at doi:10.1101/gad.203406.112

Supplemental
Material $\quad$ http://genesdev.cshlp.org/content/suppl/2013/01/24/27.2.157.DC1

References This article cites 37 articles, 11 of which can be accessed free at: http://genesdev.cshlp.org/content/27/2/157.full.html\#ref-list-1

License

Email Alerting Receive free email alerts when new articles cite this article - sign up in the box at the top Service right corner of the article or click here.

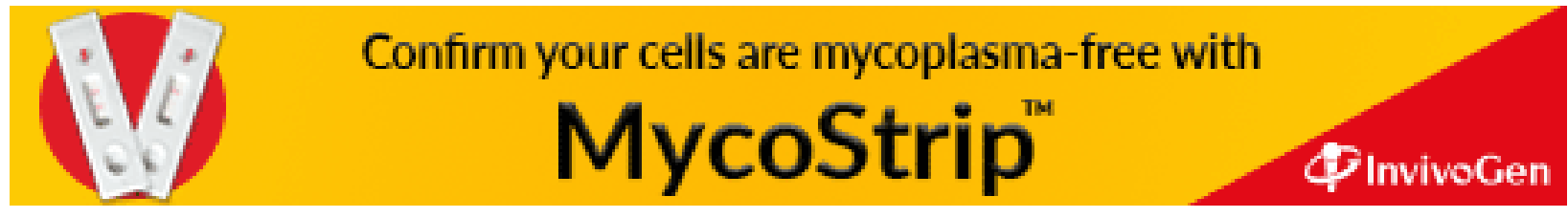

